\title{
Estimativa do índice de área foliar e determinação do coeficiente de extinção luminosa da abóbora Cucurbita moschata var. japonesa
}

\author{
Estimation of leaf area index and determination of light \\ extinction coefficient of pumpkin Cucurbita moschata \\ var. japanese \\ Eduardo Domingos Grecco $^{1 *}$; Luiz Flávio Vianna Silveira ${ }^{2}$; \\ Victor Luiz de Souza Lima², José Eduardo Macedo Pezzopane ${ }^{1}$
}

\begin{abstract}
RESUMO
Os métodos indiretos para a determinação do índice de área foliar (IAF), que são caracterizados em medidas da radiação total que penetram no interior da cobertura vegetal, são atualmente os mais utilizados, por serem mais práticos. O estudo teve como objetivo medir e estimar o índice de área foliar, ajustar uma equação através do produto de (comprimento x largura x área foliar) e determinar o coeficiente de extinção luminosa em abóbora Cucurbita moschata var. japonesa. Para desenvolver um modelo, foram medidas vinte folhas determinando-se o comprimento e a largura e para a estimativa do índice de área foliar, foram utilizados cinco repetições de $1 \mathrm{~m}^{2}$, sendo feitas três medidas no interior das folhas e uma medida na parte superior das plantas. Posteriormente, suas folhas foram coletadas para medições de área foliar em laboratório. Para a obtenção do coeficiente de extinção luminosa (k) foram medidos quinze pontos da cultura, em condições de sol e de sombra. O IAF medido foi 1,145 e o estimado 2,624, mostrando um modelo matemático com crescimento linear originando uma reta e o coeficiente de extinção foi de 0,59 . Este trabalho foi importante para determinar os valores de IAF medido, estimado e o coeficiente de extinção para a cultura de abóbora.
\end{abstract}

Palavras-chave: Cucurbita moschata, Índice de área foliar, Método indireto.

\begin{abstract}
The indirect methods for the determination of leaf area index (LAI), which are characterized in total that measures radiation penetrate inside the cover, are currently the most used, because they are more practical. The study was to measure and estimate the leaf area index, adjust an equation through product (length $x$ width $x$ leaf area) and determine the light extinction coefficient in pumpkin Cucurbita moschata var. japanese. To develop a model, twenty sheets were measured by calculating the length and width and to the estimation of leaf area index were used five repetitions of $1 \mathrm{~m}^{2}$, being made three measures inside the leaves and a measure at the top of the plants. Later, its leaves were collected for leaf are a measurements in the laboratory. For obtaining the light extinction coefficient ( $k$ ) were measured fifteen points of culture, in conditions of sun and shade. The LAI was measured and estimated 2.624 and 1.145, showing a mathematical model with linear growth and yields a straight and the extinction coefficient was 0.59. This work was important to determine the values of LAI measured, estimated and the extinction coefficient for the cultivation of pumpkin.
\end{abstract}

Key words: Cucurbita moschata, Leaf area index, Indirect method.

As cucurbitáceas apresentam uma vasta possibilidade de usos, desde o consumo in natura até a produção de industrializados, gerando emprego e renda em diversos Estados do Brasil. Dentre as espécies mais cultivadas desta família podemos destacar a melancia (Citrullus lanatus), abóbora (Cucurbita moschata), abobrinha (Cucurbita pepo) e moranga (Cucurbita maxima), que ocupam uma parcela significativa do agronegócio brasileiro. (Pesagro, 2005; Seagri, 2005), havendo a necessidade cada vez maior de estimar a capacidade produtiva das culturas, em diferentes condições do meio, tanto

1 Programa de Pós-Graduação em Produção Vegetal. CCA-UFES, Alto Universitário S/N, CEP: 29500-000, Alegre, ES. E-mail: grecco.eduardo@hotmail.com

2 Programa de Pós-Graduação em Produção Vegetal - UENF/CCTA. Av. Alberto Lamego, 2000 - Horto, CEP: 28013-602, Campos dos Goytacazes, RJ. E-mail: viannasilveira@hotmail.com; tydin@hotmail.com

* Autor para correspondência. 
para estimativa de safras, preparo de sua colheita, armazenagem e escoamento, como para estimar os possíveis efeitos de alterações nas técnicas de produção e de aumento de produtividade.

Para suprir essa necessidade, modelos agrometeorológicos e de simulação de cultivos estão sendo aprimorados (Müller et al., 2005). Os métodos diretos para determinar o índice de área foliar (IAF) não são facilmente utilizados, pois necessitam de um trabalho dispendioso e da destruição de uma grande quantidade de vegetação, como os experimentos em culturas florestais. Neste sentido, os métodos indiretos, que são caracterizados em medidas da radiação total que penetram no interior da cobertura vegetal, são atualmente os mais utilizados (Mcwilliam et al., 1993). Na determinação do IAF, estes métodos indiretos de radiação apresentam uma forma muito mais prática de obtenção dos dados, além de produzir resultados próximos aos dos métodos diretos (Roberts et al., 1996).

A eficiência na interceptação e absorção de radiação fotossinteticamente ativa (PAR) está diretamente relacionada à organização espacial das folhas, representada pela densidade de cobertura vegetal, distribuição horizontal e vertical de folhas e o ângulo de inserção foliar (Moreira, 2005). O ângulo de inserção foliar, juntamente com o (IAF), regulam o grau de penetração da radiação no interior da copa da planta utilizando um coeficiente de extinção luminosa $(\mathrm{K})$ descrito por Monsi e Saeki (1953) citado por Hirose (2005). No entanto, sabe-se que o coeficiente de extinção é dependente tanto da arquitetura foliar como do ângulo zenital do Sol, apresentando, portanto, grande variação em função das variedades e também do horário do dia. Os valores de taxa de crescimento da cultura variam de acordo com a morfologia da planta e com o k (Sheehy \& Cooper, 1973).

Alguns pesquisadores têm investigado a influência do déficit hídrico sobre a eficiência fotossintética de algumas culturas. O déficit hídrico que ocorre frequentemente em regiões áridas reduz as trocas gasosas, especialmente de $\mathrm{CO}_{2}$, e sua condução para a folha (Lopes et al., 1988). Assim as características morfológicas e fisiológicas das plantas determinam suas habilidades competitivas pela água do solo (Procopio et al., 2004).

As plantas estão sujeitas a uma grande variedade de estresses ambientais, incluindo temperaturas inadequadas, condições físico-químicas de solo desfavoráveis e várias doenças e pragas. Contudo, pode-se dizer que o déficit hídrico reduz o crescimento e a produtividade vegetal mais que todos os outros estresses combinados, pois ocorre em qualquer local, mesmo nas regiões consideradas úmidas (Kramer, 1983). Para Taiz \& Zeiger (1991), o déficit hídrico não é limitado apenas às regiões áridas e semi-áridas. Segundo observaram, mesmo em regiões consideradas climaticamente úmidas, a distribuição irregular das chuvas provoca períodos cuja disponibilidade hídrica limita o crescimento das plantas.

O objetivo deste estudo foi medir e estimar o índice de área foliar, ajustar uma equação através do produto de (comprimento $\mathrm{x}$ largura $\mathrm{x}$ área foliar) e o k em Cucurbita moschata var. japonesa.

As medidas foram realizadas na área experimental do Centro de Ciências Agrárias da Universidade Federal do Espírito Santo em cultura de abóbora japonesa. Para desenvolver um modelo linear que relaciona o comprimento e largura das folhas com a área foliar, foram medidas vinte folhas determinando-se o comprimento e a largura através de régua e a área foliar correspondente foi medida com o equipamento LI-COR LI-3100C. Os resultados obtidos foram submetidos à análise de variância e ajustados pela equação de regressão linear. Os dados foram analisados no programa Sigma Plot (SPSS, 2001b).

Para a estimativa do índice de área foliar, foram utilizados cinco repetições com medida de $1 \mathrm{~m}^{2}$ através de um quadrante de madeira. A medida da radiação para a estimativa do IAF foi feito pelo equipamento LAI-2000, sendo feitas três medidas no interior das folhas e uma na parte superior das plantas. Posteriormente, as folhas foram coletadas e armazenadas em sacos de papel para posterior medição de área foliar que foi realizada uma hora após a coleta.

As medições de área foliar foram feitas no equipamento LI-COR LI-3100C, no Laboratório do Núcleo de Difusão de Tecnologia e Recursos Hídricos (NEDTEC/CCA-UFES), no município de Jerônimo Monteiro-ES. Para a obtenção do k foram feitas coletas de dados em 15 pontos da cultura, em condições de sol (acima do dossel) e de sombra (abaixo do dossel), com o equipamento LI-COR line (quantum sensor) modelo LI-250. O IAF utilizado para calcular o $\mathrm{k}$ foi calculado com o equipamento LI-COR LI-3100C. 
Com os dados do IAF e interceptação de luz, calculou-se o k, conforme descrito por Sheehy e Cooper (1973) sendo o $\mathrm{k}=\left[\log \mathrm{e}\left(\mathrm{I} / \mathrm{I}_{0}\right)\right] / \mathrm{IAF}$, onde I e $\mathrm{I}_{0}$ são os valores de irradiância abaixo e acima da folhagem, respectivamente. Os resultados do IAF medido foram de 1,145 e o estimado 2,624. Este valor acima pode ser explicado pelo horário no qual as análises foram feitas, tendo uma alta incidência de luminosidade.

O modelo matemático baseado no (comprimento $\mathrm{x}$ largura $\mathrm{x}$ área foliar) mostrou um crescimento linear originando uma reta, comprovando que a equação originada é adequada para explicar a relação entre as variáveis, onde o coeficiente de determinação $\left(\mathrm{R}^{2}\right)$ explicou mais de $90 \%$ dos resultados apresentados (Figura 1). Observou-se uma elevada correlação entre o comprimento e largura das folhas, indicando que as folhas das plantas cujas copas encontravam-se em condições de sombra apresentaram maior área foliar individual. Na Figura 2 observou-se uma grande dispersão dos pontos, diferenciando dos resultados encontrados para o IAF em laboratório, o que pode ser explicado pelo excesso de nebulosidade no dia em que foram feitas as medidas. A área foliar foi maior em plantas sob baixa luminosidade, indicando que o aparato fotossintético desta espécie sofreu um ajustamento.

Podemos observar que na Figura 3 a relação dos pontos de sol e sombra foram menores na sombra pela barreira física que as folhas proporcionam a entrada desta radiação sob o dossel da planta. Com estes valores de radiação e do IAF medido foi calculado o coeficiente de extinção que apresentou um valor de 0,59. Segundo Madakadze et al. (1998) a maioria dos trabalhos relata que os valores de $\mathrm{k}$ variam entre 0 e 1 .

As folhas de sombra são em geral menos espessas, apresentam menor peso foliar específico, menor relação entre parênquima paliçádico e lacunoso e maior área foliar (Nobel, 1991). Estas folhas de sombra poderiam ter outra fonte de luz direta, como, por exemplo, fachos de luz que disponibilizam luz no comprimento de onda vermelho e parecem permitir que houvesse uma transmitância de luz azul maior do que a esperada. A adaptação às baixas luminosidades é uma característica genética, a qual faz com que as folhas apresentem estrutura anatômica e propriedades fisiológicas

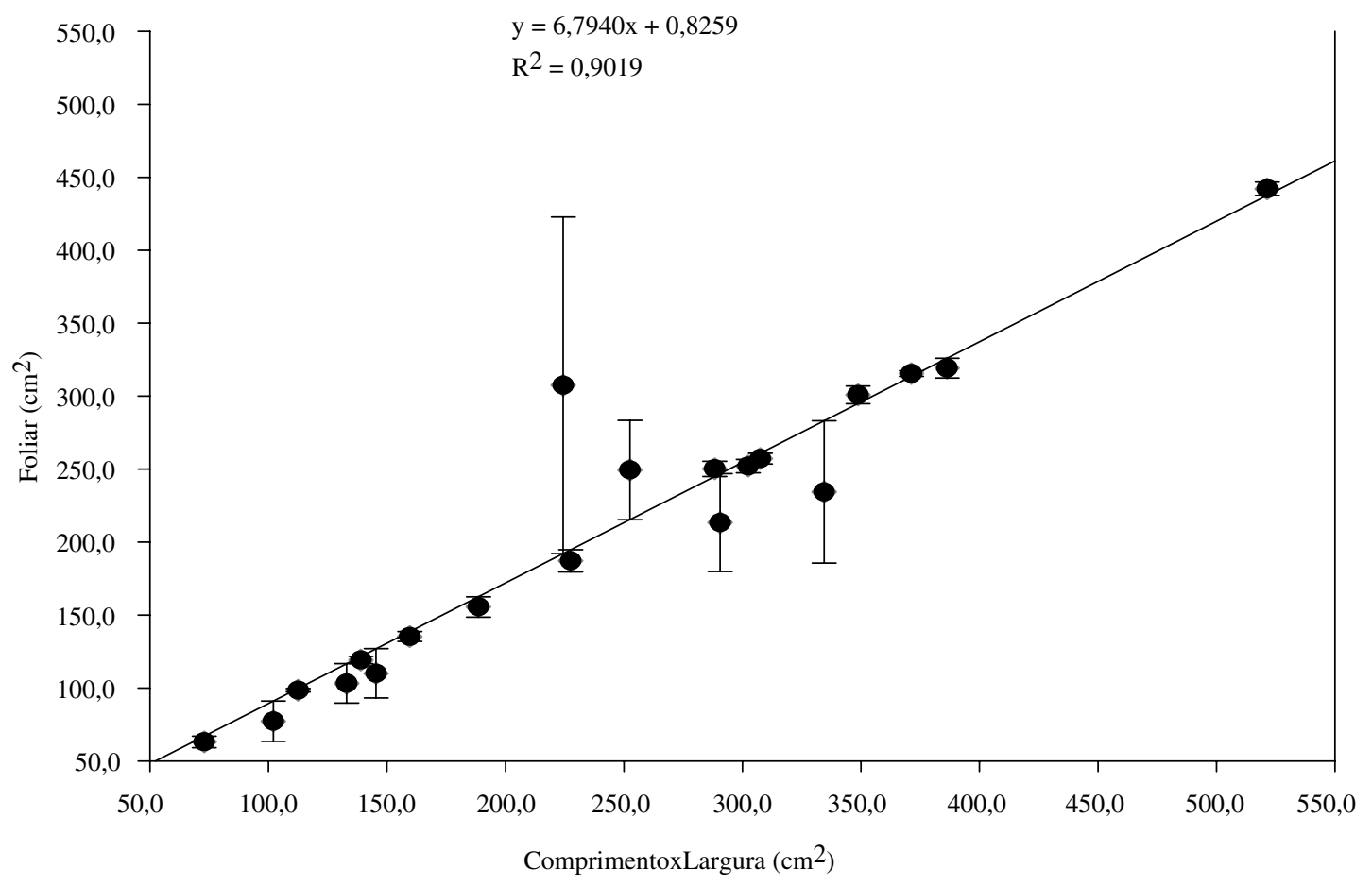

Figura 1. Modelo baseado no comprimento*largura*área foliar. 


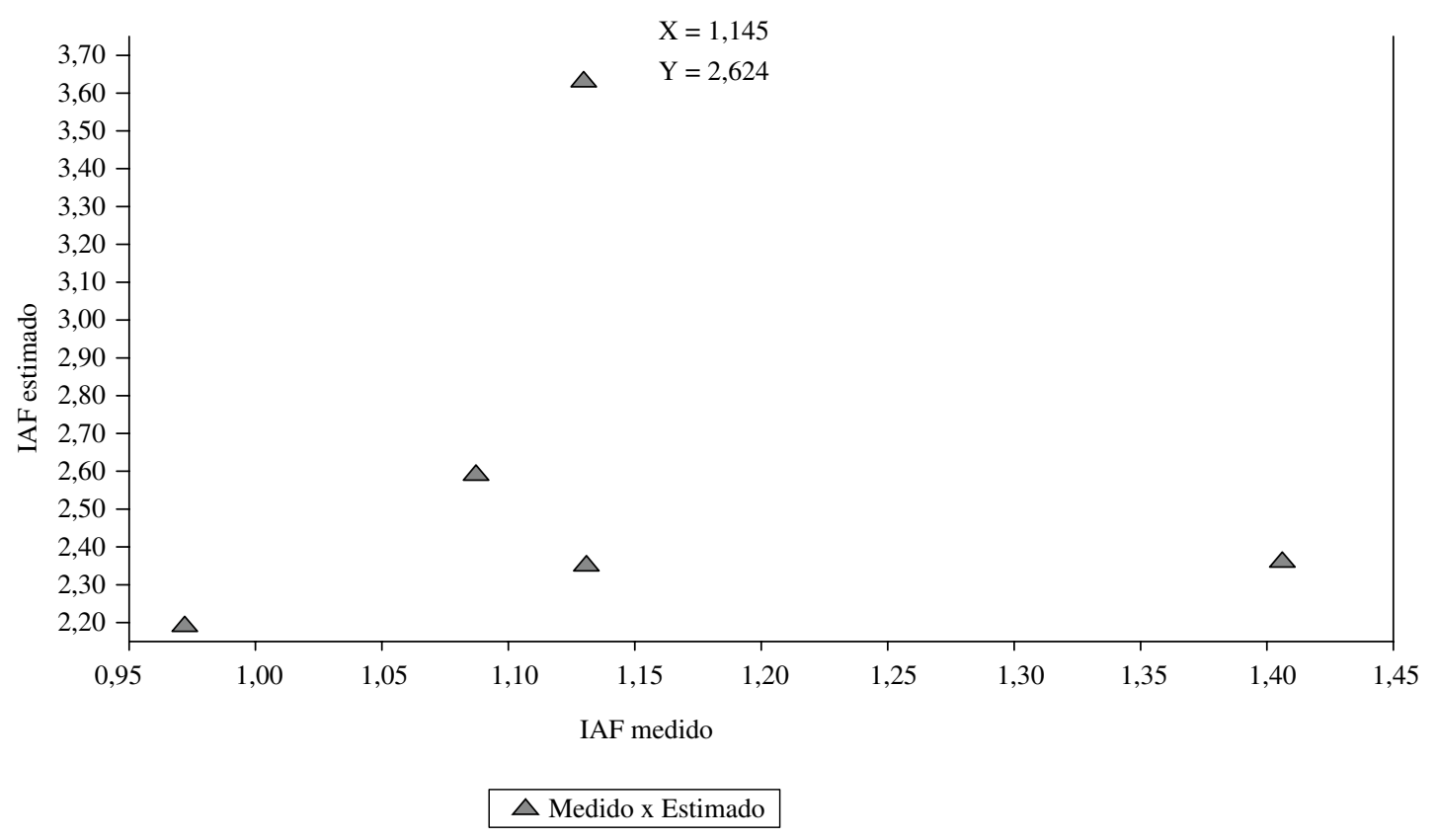

Figura 2. Comparação do IAF estimado e medido.

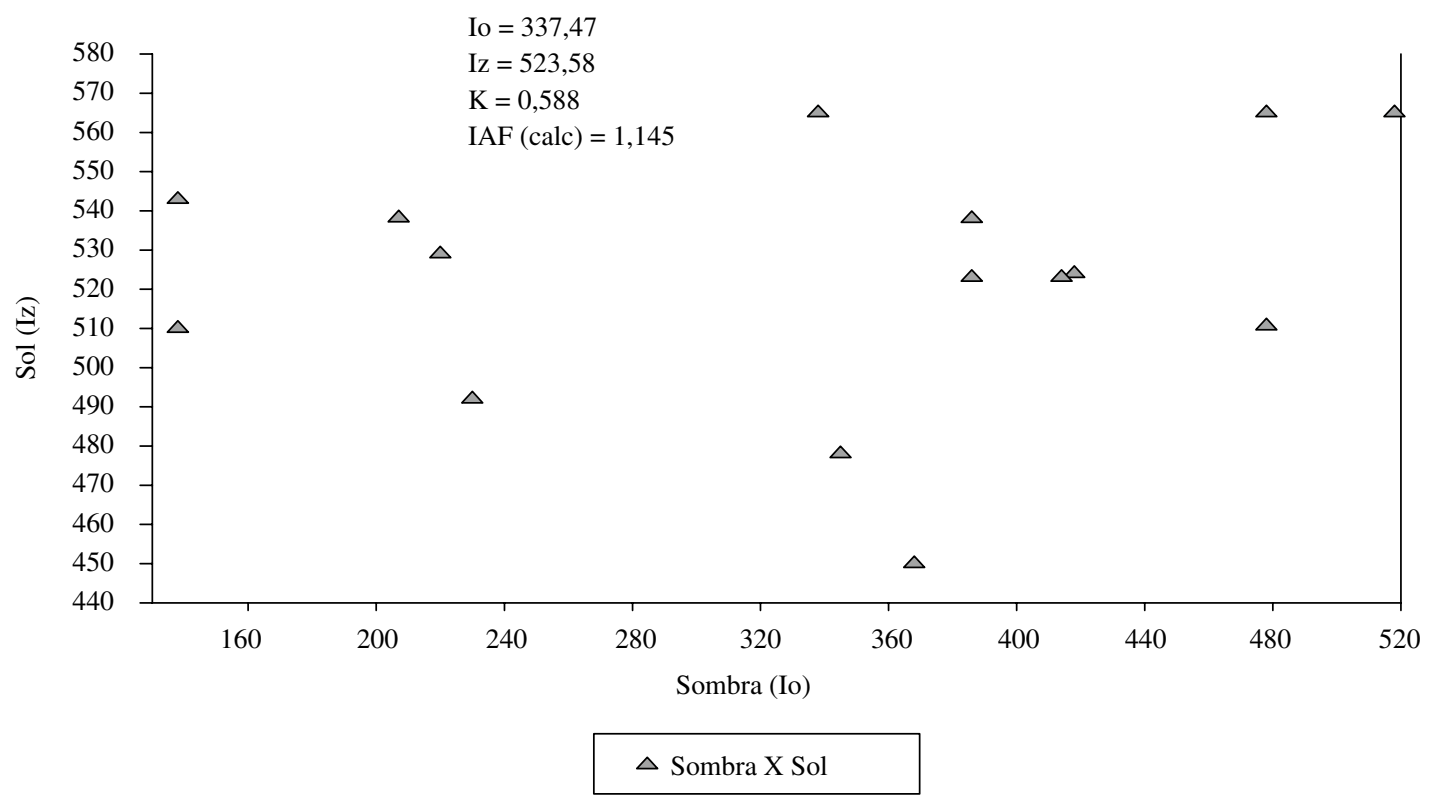

Figura 3. Valores de interceptação de luz acima e abaixo do dossel.

que as capacitem ao uso efetivo da radiação solar disponível (Larcher, 2000).

O regime de radiação solar não é diferente entre as folhas de sombra e sol, o que implica que as plantas de sub-bosque e aquelas em pleno sol, devem estar aclimatadas aos respectivos regimes de radiação solar fotossinteticamente ativa. Plantas localizadas em climas áridos e semi-áridos possuem folhas mais espessas e caracterizam pela resistência estomática. $\mathrm{O}$ aumento no número de estômatos $/ \mathrm{mm}^{2}$ geralmente é observado em folhas de plantas expostas ao sol, como visto por 
vários autores (Almeida et al., 2004), e isto pode ser um indicativo de um mecanismo de adaptação das plantas às condições de baixa disponibilidade hídrica no solo.
Este estudo foi importante para determinar os valores de IAF medido e estimado e o $\mathrm{k}$ através de equipamentos modernos que mostram os resultados instantaneamente para a cultura da abóbora japonesa.

\section{Literatura Citada}

Almeida, L. P.; Alvarenga, A. A.; Castro, E. M.; Zanela, S. M.; Vieira, C. V.

2004 Crescimento inicial de plantas de Cryptocaria aschersoniana Mez. submetidas a níveis de radiação solar. Ciência Rural, Santa Maria, 34 (1): 83-88.

Hirose, $T$.

2005 Development of the Monsi-Saeki theory on canopy structure and function. Annals of Botany. 95: 483-494.

Kramer, P.J.

1983 Water relations of plants. New York: Academic Press, $489 \mathrm{p}$.

Larcher, W.

2000 Ecofisiologia vegetal. São Carlos: RiMa Artes e Textos, $531 \mathrm{p}$.

Lopes, B. F.; Seter, T. L.; McDavid, C. R.

1988 Photosynthesis and water vapor exchange of pingeonpea leaves in response to water deficit and recover. Crop Science. Madison, 28: 141-145.

Madakadze, I. C.; Coulman, B. E.; Peterson, P.; Stewart, K. A.;

Samson, R.; Smith, D. L.

1998. Leaf area development, light interception, and yield among switchgrass populations in a short-season area. Crop Science, 38 (3): 827-834.

McWillian, A.L.C.; Roberts, J.M.; Cabral, M.R.; Leitão,

M.V.B.R.; Costa, C.L.; Maitelli, G.T.; Zamparonl, C.A.

1993 Leaf Area Index and above ground biomass of terra firme rain Forest and adjacent clearings. In.: Amazônia. Functional Ecology, 7: 210-217.

Monsi, M.; Saeki, T.

1953 Uber den Lichfaktor in den Pflanzengesellschaften und seine Bedeuttung fur die Stoffproduktion. Jardin Botanique, Japon, 14: 22-52.

Moreira, M. A.

2005 Fundamentos do sensoriamento remoto e metodologias de aplicação, $3^{\mathrm{a}}$ ed., Viçosa, $320 \mathrm{p}$.
Müller, A.G.; Bergamaschi, H.; Bergonci, J.I.; Radin, B.; França, S.; Guilardi, M.I.S.

2005 Estimativa do índice de área foliar do milho a partir da soma de graus-dia. Revista Brasileira de Agrometeorologia, Santa Maria, 13 (1): 65-71.

Nobel, P.S

1991 Physioquemical and environmental plant physiology. Academic Press, San Diego.

Pesagro.

2005 A cultura do maxixe (Cucumis anguria L.). Disponível em <http://www.pesagro.rj.gov.br/difusao/infotec/maxixe. htm>, consultado em 15/01/11.

Procopio, S.O.; Santos, J.B.; Silva, A.A.; Donagema, G.K.; Mendonça, E.S.

2004 Ponto de murcha permanente de soja, feijão e plantas daninhas. Planta daninha, 22 (1): 35-41.

Roberts, J.M.; Cabral, O.M.R.; Da Costa, J.P.; McWilliam, A.L.C.; De Sá, T.D.

1996 An overview of the leaf area index and physiological measurements during ABRACOS. In: Amazonian Deforestation and Climate, pp. 287-306.

SEAGRI

2005 Cultivo de cucurbitáceas, disponível em <http://www. seagri.ba.gov.br>, consultado em 15/01/11.

Sheehy, J.E.; Cooper, J.P.

1973 Light interception, photosynthetic activity, and crop growth rate in canopies of six temperate forage grasses. Journal of Applied Ecology, Oxford, 10 (1): 239-250.

SPSS

2001b Sigma Plot 2002 for Windows version 8.0, SPSS Inc., Chicago.

Taiz, L.; Zeiger, E.

2009 Fisiologia Vegetal, 4a ed. Porto Alegre, 848 p. 
